Int. J. Electrochem. Sci., 15 (2020) 9037 - 9048

International Journal of

ELECTROCHEMICAL

SCIENCE

www.electrochemsci.org

\title{
Simultaneous Detection of Morphine and Diclofenac Using Graphene Nanoribbon Modified Screen-Printed Electrode
}

\author{
Peyman Mohamadzadeh Jahani ${ }^{1}$, Sayed Zia Mohammadi ${ }^{2}$, Ali Khodabakhshzadeh ${ }^{1}$, Joo Hwan Cha ${ }^{3}$, \\ Mehdi Shahedi Asl ${ }^{4}$, Mohammadreza Shokouhimehr ${ }^{5}$, Kaiqiang Zhang ${ }^{6, *}$, Quyet Van Le ${ }^{7, *}$, \\ Wanxi Peng ${ }^{8,9, *}$ \\ ${ }^{1}$ Department of Basic Sciences, School of Medicine, Bam University of Medical Sciences, Bam Iran \\ ${ }^{2}$ Department of Chemistry, Payame Noor University, Tehran, Iran \\ ${ }^{3}$ Innovative Enterprise Cooperation Center, Korea Institute of Science \& Technology, Hwarangro 14- \\ gil, Seongbuk-gu, Seoul, Korea \\ ${ }^{4}$ Marine Additive Manufacturing Centre of Excellence (MAMCE), University of New Brunswick, \\ Fredericton, NB, E3B 5A1, Canada \\ ${ }^{5}$ Department of Materials Science and Engineering, Research Institute of Advanced Materials, Seoul \\ National University, Seoul 08826, Republic of Korea \\ ${ }^{6}$ Jiangsu Key Laboratory of Advanced Organic Materials, Key Laboratory of Mesoscopic Chemistry \\ of MOE, School of Chemistry and Chemical Engineering, Nanjing University, Nanjing, Jiangsu \\ 210023, China \\ ${ }^{7}$ Institute of Research and Development, Duy Tan University, Da Nang 550000, Vietnam \\ ${ }^{8}$ College of Forestry, Henan Agricultural University, Zhengzhou 450002, China \\ ${ }^{9}$ School of Automotive Engineering, Huanghe Jiaotong University, Jiaozuo 454950, China \\ *E-mail: kaiqiangzhang126@126.com, Levanquyet@dtu.edu.vn, pengwanxi@163.com
}

doi: $10.20964 / 2020.09 .14$

Received: 13 April 2020 / Accepted: 25 June 2020 / Published: 10 August 2020

The graphene nanoribbon modified screen printed electrode (G/SPE) has been produced to determine morphine and diclofenac via differential pulse voltammetric, cyclic voltammetric, and chronoamperometry. Graphene nanoribbon shows great selectivity and sensitivity in determining morphine and diclofenac. The impact of scan rates has been explored. Findings showed that it is diffusion-controlled. The impact of morphine concentrations has been investigated in ranges of 0.07600.0 $\mu \mathrm{M}$. Determination limit for morphine has been $20.0 \mathrm{nM}$. In general, an easy experimental method for manufacturing graphene oxide nanoribbon has been suggested that takes advantage of selectivity, reproducibility, and sensitivity toward electro-active specimens, as well as biological matrices.

Keywords: Morphine,Diclofenac, Graphite Screen Printed Electrode, Graphene nanoribbon. 


\section{FULL TEXT}

(C) 2020 The Authors. Published by ESG (www.electrochemsci.org). This article is an open access article distributed under the terms and conditions of the Creative Commons Attribution license (http://creativecommons.org/licenses/by/4.0/). 